\title{
Conditionals, indeterminacy, and triviality
}

\author{
Justin KHOO \\ jkhoo@mit.edu \\ Forthcoming in Philosophical Perspectives (please cite published version)
}

This paper is about some obstacles to relating the probabilities of indicative conditionals to their truth conditions. To get a feel for the issue, consider the following plausible principle connecting the probability that a sentence is true with its truth conditions:

Sentential probability: The probability that a declarative sentence $\mathrm{p}$ is true in some context $c$ equals the probability of $p$ (in $c$ ), where $p$ is the proposition p expresses in $c$.

Add in the following well-confirmed observation about the probabilities of indicative conditionals: ${ }^{1}$

Ramsey's observation: many indicative conditional/context pairs $\langle\ulcorner$ if p, q $\urcorner, c\rangle$ are Ramseyan.

- Where $\langle\ulcorner$ if p, q $\urcorner, c\rangle$ is Ramseyan iff the probability that $\ulcorner$ if $\mathrm{p}, \mathrm{q}\urcorner$ is true in $c$ is equal to the conditional probability that $\mathrm{q}$ is true in $c$ given that $\mathrm{p}$ is true in $c$.

Ramsey's observation is confirmed by the fact that we typically endorse conditionals (in the sense of finding acceptable, or having a high degree of belief in) to the degree that their consequent is likely given their antecedent. For instance, the Bulls are a pretty good team, and nearly unbeatable when their star Michael Jordan suits up. As a result, though

\footnotetext{
${ }^{1}$ So-called because this equation between the probabilities of conditionals and conditional probabilities was first suggested by Frank Ramsey:

If two people are arguing 'If $\mathrm{p}$ will q?' and both are in doubt as to $\mathrm{p}$, they are adding $\mathrm{p}$ hypothetically to their stock of knowledge and arguing on that basis about q ... We can say that they are fixing their degrees of belief in q given p. (Ramsey 1931, p. 249)
} 
we're not sure whether Jordan played in last night's game, we think (1-a) is very likely. Nonetheless, we find (1-b) extremely unlikely.

a. If Jordan played last night, the Bulls won.

b. If Jordan played last night, it rained in the Sahara.

If (1-a) is Ramseyan, then the likelihood of (1-a) goes by the conditional likelihood that the Bulls won given that Jordan played last night — and the latter value is high since the Bulls almost always win when Jordan plays, so we predict that (1-a) is very likely. Furthermore, we predict if (1-b) is Ramseyan, then we predict that it is unlikely because the conditional likelihood that it rained in the Sahara given that Jordan played last night is low. ${ }^{2}$

The problem is that Ramseyan conditional/context pairs make trouble for Sentential probability given certain other auxiliary assumptions. For instance, the triviality results of David Lewis can be leveraged, along with Sentential probability, to the conclusion that a conditional/context pair is Ramseyan only if the probability of the conditional (in that context) is equal to the probability of its consequent (in that context)-we'll call problems like this arising from the various triviality proofs problems of triviality. ${ }^{3} \mathrm{~A}$ related problem charges that Sentential probability is incompatible with a particular kind of Ramseyan conditional/context pair - one whose conditional is indeterminate at any world in which its antecedent is false - and yet our intuitions confirm many such conditional/context pairs; we'll call this the problem of indeterminacy. ${ }^{4}$

These problems have motivated two radical views in the literature on conditionals - for instance, non-propositionalism, which rejects Sentential probability (see for instance Adams 1975, Gibbard 1981, Edgington 1995, Bennett 2003), and the material conditional theory, which rejects Ramsey's observation (see for instance Lewis 1976, Jackson 1979, Grice 1989). However, given the intuitive appeal of both Sentential probability and Ramsey's observation, a theory that allows us to preserve both is prima facie more plausible than a theory that rejects one or the other. But preserving both requires a theory of indicative conditionals that can handle the troublesome Ramseyan conditional/context pairs, and this requires the theory to explain how the probabilities of conditionals are

\footnotetext{
${ }^{2}$ For further confirmation of Ramsey's observation, see the discussion in Edgington 1995, Bennett 2003. However, there seem to be non-Ramseyan indicative conditional/context pairs as well-see McGee 2000, Kaufmann 2004, Rothschild 2011, Khoo 2013.

${ }^{3}$ For Lewis's original proof, see Lewis 1976, 1986. For variations, see Stalnaker 1976, Gibbard 1981, Hájek 1989, 1994, 2011b, 2012, Hájek and Hall 1994, Edgington 1995, Bennett 2003.

${ }^{4}$ For a related problem for subjunctive conditionals, see Hájek 2011a.
} 
related to their truth conditions. In this paper, I aim to motivate such a theory by arguing that we can plausibly avoid both the problems of indeterminacy and triviality by rejecting some of their auxiliary assumptions.

Here's the plan for what follows. I begin by discussing the problem of indeterminacy (§1.1-1.2). In $\S 1.3$, I sketch my favored semantics for indicative conditionals and in $\S 1.4$ show how it resolves this indeterminacy problem. In $\S 2$, I turn to the various triviality proofs, splitting them into two kinds, and show how our semantics handles the resulting two problems of triviality. Perhaps unsurprisingly, the relation between the probabilities of indicative conditionals and their truth conditions turns out to be more complex than anticipated - on the theory I defend, the truth conditions of indicatives are highly context dependent and such that an indicative conditional may be indeterminate in truth value at each possible world throughout some region of logical space and yet still have a nonzero probability throughout that region. Nonetheless, I hope to make an initial case for the claim that these extra complexities are plausible and thus no barrier to endorsing both Sentential probability and Ramsey's observation.

\section{The problem of indeterminacy}

Roughly, the problem of indeterminacy charges that Sentential probability is incompatible with Ramseyan conditional/context pairs whose conditional is indeterminate in truth value at any world in which its antecedent is false. This is a problem for Sentential probability because, intuitively, there are many such conditional/context pairs. In order to formally state the problem of indeterminacy and how I propose to solve it, it will be helpful to review a few technical preliminaries. Throughout, I'll use lowercase roman letters p, q, $\mathrm{r}, \ldots$ to denote sentences, and italicized letters $p, q, r, \ldots$ to denote the propositions expressed by the corresponding sentences (in the relevant context-I'll leave that parameter unvoiced unless it's necessary to keep track of). I will be assuming that indicative conditionals express propositions throughout in order to defend Sentential probability - so, for a conditional/context pair $\langle\ulcorner$ if p, $\mathrm{q}\urcorner, c\rangle$, we'll let if $p, q$ denote the proposition expressed by $\ulcorner$ if $\mathrm{p}, \mathrm{q}\urcorner$ at the context $c$. Allowing for a certain degree of idealization, we'll assume that an agent's credences can be represented by a precise probability function $P$ from propositions to values in $[1,0]$, and assume that propositions are simply sets of possible worlds. Thus, we can define a probability function $P$ as any function from subsets of $W$ (the propositions) to values in $[1,0]$ obeying the Kolmogorov axioms: 
1. $P(p) \geq 0$, for all $p \in \wp(W)$

2. $P(W)=1$

3. $P(p \vee q)=P(p)+P(q)$, for all $p, q \in \wp(W)$ such that $p \wedge q=\emptyset$

We add to [1]-[3] the following (standard) definition of conditional probability, setting aside for now worries that this will not serve as a definition but at best an approximation of the intuitive notion (characterized above): ${ }^{5}$

4. $P(q \mid p)=\frac{P(p \wedge q)}{P(p)}$, where $P(p)>0$

Sentential probability is a claim connecting the probability that a sentence is true with the probability of the proposition that sentence expresses. We formalize sentential probability as follows. Let Pr be a function from contexts and sentences to values in $[1,0]$ which give the probability that that sentence is true in that context (and a corresponding conditional sentential probability function that takes contexts and two sentences into $[1,0]$ defined in a way analogous to [4]). We can now restate what it is for a conditional/context pair to be Ramseyan:

(2) A conditional/context pair $\langle\ulcorner$ if $\mathrm{p}, \mathrm{q}\urcorner, c\rangle$ is Ramseyan iff $\operatorname{Pr}_{c}(\ulcorner$ if $\mathrm{p}, \mathrm{q}\urcorner)=\operatorname{Pr}_{c}(\mathrm{q} \mid \mathrm{p})$. along with our principles from the introduction:

Sentential probability: For any $\mathrm{p}$ and $c, \operatorname{Pr}_{c}(\mathrm{p})=P_{c}(p)$.

(Where $P_{c}$ is the probability function of the relevant agent in $c$ and $p$ the proposition expressed by $\mathrm{p}$ at $c$.)

Ramsey's observation: for many $\langle\ulcorner$ if $\mathrm{p}, \mathrm{q}\urcorner, c\rangle, \operatorname{Pr}_{c}(\ulcorner$ if $\mathrm{p}, \mathrm{q}\urcorner)=\operatorname{Pr}_{c}(\mathrm{q} \mid \mathrm{p})$.

Notice that, given Sentential probability, it follows that:

A conditional/context pair $\langle\ulcorner$ if $\mathrm{p}, \mathrm{q}\urcorner, c\rangle$ is Ramseyan iff $P_{c}($ if $p, q)=P_{c}(q \mid p)$.

Since we are assuming Sentential probability in what follows, we'll often move freely between $\operatorname{Pr}_{c}$ and $P_{c}$. Next, let's define two important properties of conditional/context pairs:

\footnotetext{
${ }^{5}$ For problems with this definition, see Hájek 2003. We'll ignore these for now.
} 
$\langle\ulcorner$ if $\mathrm{p}, \mathrm{q}\urcorner, c\rangle$ is probability centered iff $\operatorname{Pr}_{c}(\ulcorner$ if $\mathrm{p}, \mathrm{q}\urcorner \mid \mathrm{p})=\operatorname{Pr}_{c}(\mathrm{q} \mid \mathrm{p})$.

$$
\langle\ulcorner\text { if } \mathrm{p}, \mathrm{q}\urcorner, c\rangle \text { is antecedent independent iff } \operatorname{Pr}_{c}(\ulcorner\text { if } \mathrm{p}, \mathrm{q}\urcorner \mid \mathrm{p})=\operatorname{Pr}_{c}(\ulcorner\text { if } \mathrm{p}, \mathrm{q}\urcorner) \text {. }
$$

Notice that if $\langle\ulcorner$ if $\mathrm{p}, \mathrm{q}\urcorner, c\rangle$ is both probability centered and antecedent independent, then it is Ramseyan. In the next section, I'll argue that indicatives are always probability centered:

PC: every conditional/context pair is probability centered.

However, I won't argue that every conditional/context pair is antecedent independent, since I think this is false, and furthermore that the exceptional cases are responsible for non-Ramseyan conditional/context pairs (see the discussion in McGee 2000, Kaufmann 2004, Rothschild 2011, Khoo 2013). Let's turn now to the motivation for PC. In $\S 1.2$, I'll draw on PC to motivate the problem of indeterminacy.

\section{$1.1 \quad \mathrm{PC}$}

Although my motivation for PC relies on Sentential probability, nothing crucial turns on this assumption - we can reformulate the arguments in terms of probabilistic validity on sentences instead of classical validity on propositions. ${ }^{6}$ PC follows from Strong centering, which itself follows from Conditional excluded middle (given the validity of modus ponens):

Strong centering: for any $\langle\ulcorner$ if $\mathrm{p}, \mathrm{q}\urcorner, c\rangle: \models p \supset(q \equiv$ if $p, q)$

Conditional excluded middle (CEM): for any $\langle\ulcorner$ if p, $q\urcorner, c\rangle$ and $\langle\ulcorner$ if p, not- $q\urcorner$, $c\rangle:=($ if $p, q) \vee($ if $p, \neg q)$

Basically, Strong Centering ensures that if $p$ is true, then if $p, q$ is true iff $q$ is true. CEM ensures that, for any conditional and context, either the proposition expressed by the conditional or the proposition expressed by that conditional's negated consequent cousin is true. Here's why CEM + modus ponens entails Strong Centering. Suppose CEM holds; then if $p$ and $q$ are both true, if $p, q$ must be true ${ }^{7}$ - and furthermore, if $p$ is true and

\footnotetext{
${ }^{6}$ See Adams 1975, 1998, for instance. I should point out that both Edgington, a non-propositionalist, and Kaufmann, a non-classical theorist, endorse Strong centering - see Edgington 1995, p. 290 and Kaufmann 2005, p. 199.

${ }^{7}$ This follows given CEM by the following line of reasoning. Suppose $p, q$, and if $p, \neg q$ are true. Then $\neg q$ is true by modus ponens. But this is a contradiction. So if $p, \neg q$ is false. But then by CEM if $p, q$ is true.
} 
if $p, q$ is true, then $q$ is true by modus ponens. Here's why Strong Centering entails PC. Suppose Strong Centering holds; then if $p, q$ is true at exactly the $q$-worlds in which $p$ is true. Hence, conditional on $p$, the probability of if $p, q$ equals the probability of $q$. Therefore, for any $\langle\ulcorner$ if $\mathrm{p}, \mathrm{q}\urcorner, c\rangle: P_{c}($ if $p, q \mid p)=P_{c}(q \mid p)$-and this is just PC.

Thus, evidence for either Strong centering or CEM is evidence supporting PC. And, intuitively, both of the former principles are intuitively supported. Take Strong centering first. Suppose John recently rolled a fair six-sided die and kept the result hidden, and that we place a bet on the outcome of the roll. I bet that if John rolled a prime, then he rolled an odd, and you bet against me. Intuitively, if John rolled an odd prime (a 3 or 5 ) then I win the bet, while if John rolled an even prime (a 2) then you win the bet (set aside what happens if John doesn't roll a prime for now). If our betting intuitions are evidence for the truth conditions of (6), then it seems that $p \wedge q$ is sufficient for the truth of if $p, q$, while $p \wedge \neg q$ is sufficient for the falsity of if $p, q$.

(6) If John rolled a prime then he rolled an odd.

That is, our betting intuitions confirm Strong centering. ${ }^{8}$

Next, CEM is supported by the fact that negations of conditionals are often interpreted as conditionals with negated consequents: ${ }^{9}$

I doubt that if John rolled a prime, he rolled an even. $\approx$ I think that if John rolled a prime, he rolled an odd.

\footnotetext{
${ }^{8}$ Some may be tempted by the thought that if $p, q$ is false if it's possible that $p \wedge \neg q$, since a bare assertion of (6) seems unwarranted if it's possible that John rolled an even prime. However, this thought is at odds with the way (6) embeds. For instance, as Moss 2012 points out, (i-a) sounds much more felicitous than (i-b):

(i) a. It's more likely than not that if John rolled a prime then he rolled an odd.

b. $\quad$ \#It's not the case that if John rolled a prime then he rolled an odd.

Intuitively, (i-a) is true in $c_{1}$, and it would be odd to utter (i-b) in $c_{1}$ (just as it would be odd to utter (6) in such a context). However, a theory that predicts that (6) is falsified by the fact that it is epistemically possible that John rolled an even prime also predicts that (i-a) is false (as uttered in $c_{1}$ ) and has no explanation for why it would be odd to utter (i-b) in such a context (since it predicts that (i-b) is both true and easily knowable in such a context). An alternative account of the intuition that we ought not assert (6) if it's possible that John rolled an even prime is that, in such a case, (6) (or the proposition it expresses) would not be known and hence not satisfy the plausible knowledge norm of assertion (cf. Williamson 1996, DeRose 2002).

${ }^{9}$ I borrow this argument for CEM from Rothschild 2011.
} 
Similarly, denials of conditionals in dialogues seem equivalent to affirmations of their negated-consequent-cousins: ${ }^{10}$

(8) A: If John rolled a prime, then he rolled an even.

B: That's false, if John rolled a prime, then he rolled an odd.

Furthermore, CEM allows us to predict the equivalence of:

a. No student will succeed if he goofs off.

b. Every student will fail if he goofs off.

without failures of compositionality. ${ }^{11}$ It seems there is an abundance of evidence in favor of both CEM and Strong centering, and hence PC. ${ }^{12}$ Thus, I will take PC as established from now on. We turn now to the problem of indeterminacy.

\subsection{Stating the problem}

The problem of indeterminacy is that Sentential probability is incompatible with the existence of conditional/context pairs $\langle\ulcorner$ if p, q,$c\rangle$ that are (i) Ramseyan, (ii) such that if $p, q$ is neither true nor false at any world in which $p$ is false, and (iii) such that $\operatorname{Pr}_{c}(\mathrm{p})$ $<1^{13}$ Yet, intuitively, there are such conditional/context pairs. To begin, let's define some properties of conditional/context pairs to facilitate the presentation of the problem.

$\langle\ulcorner$ if $\mathrm{p}, \mathrm{q}\urcorner, c\rangle$ is $A$-indeterminate iff for every epistemically accessible $\neg p$-world $w$ : if $p, q$ is neither true nor false at $w$.

$\langle\ulcorner$ if $\mathrm{p}, \mathrm{q}\urcorner, c\rangle$ is $A$-uncertain iff $\operatorname{Pr}_{c}(\mathrm{p})<1$.

The first premise is the indeterminacy observation:

\footnotetext{
${ }^{10}$ It is possible to deny a conditional without affirming its negated-consequent counterpart, as in:

(i) A: If John rolled a prime, then he rolled an even.

B: No, if John rolled a prime, then he might have rolled an odd.

However, such denials typically require some emphasis on "might", which suggests metalinguistic negation is in play-cf. Horn 1985.

${ }^{11}$ See Higginbotham 1986, 2003, von Fintel and Iatridou 2002, Klinedinst 2010.

${ }^{12}$ Though, the case isn't closed: see Lewis 1973, Stalnaker 1980, Bennett 2003, Cross 2009, Williams 2010 for further discussion.

${ }^{13}$ This problem is similar to that raised by Hájek 2011a for subjunctive conditionals - there, he argues that all subjunctive conditionals are false (or indeterminate).
} 
(CI-1) There are conditional/context pairs which are Ramseyan, A-indeterminate, and A-uncertain.

Recall our example from $\S 1.1$ - suppose the context $c_{1}$ is such that John has just rolled a fair six-sided die and kept the result hidden; consider the following conditional:

If John rolled a prime, then he rolled an odd.

Intuitively, $\left\langle(6), c_{1}\right\rangle$ is Ramseyan. The conditional probability that John rolled an odd, given that he rolled a prime is $2 / 3$ since two out of the three prime outcomes $(2,3,5)$ are odd $(3,5)$. And it's extremely plausible that $\operatorname{Pr}_{c_{1}}((6))=2 / 3$. For instance, the following embedding seems true in this context:

The likelihood that if John rolled a prime, he rolled an odd is two-thirds.

Next, suppose I bet that if John rolled a prime, he rolled an odd, and you bet against me. It seems you should only accept such a bet at 2 to 1 odds - further confirmation that $\langle(6)$, $\left.c_{1}\right\rangle$ is Ramseyan. However, now consider an epistemically accessible world $w^{*}$ in which John doesn't roll a prime (a $\neg$ prime-world). Intuitively, our bet is called off at $w^{*}$ - there's no fact of $w^{*}$ that would settle the bet either way. This seems to be strong evidence that there's no fact of the matter at $w^{*}$ whether if John rolled a prime, he rolled an odd. Therefore, it seems plausible that if prime, odd is neither true nor false at $w^{*}$ - there's no fact of $w^{*}$ that could serve as its truthmaker or falsemaker. ${ }^{14}$ (For an additional argument that (6) is not false as uttered in $c$, see the discussion in fn. 8) Therefore, since we reached this conclusion by considering an arbitrary epistemically accessible $\neg$ prime-world $w^{*}$, we conclude that for any epistemically accessible $\neg$ prime-world $w$, if prime, odd is neither true nor false at $w$, and hence that $\left\langle(6), c_{1}\right\rangle$ is A-indeterminate. Finally, notice that, since we are uncertain whether John rolled a prime, $P_{c_{1}}$ (prime) $<1$. Therefore, $\left\langle(6), c_{1}\right\rangle$ is Ramseyan, A-indeterminate, and A-uncertain in $c_{1}$. Hence, (CI-1).

Next, it seems obvious that the probability that a sentence is true in a context requires at least that there are some epistemically possible worlds in that context such that the sen-

\footnotetext{
${ }^{14}$ There are at least two ways out of line of reasoning. One is that the conditional can be true at a world even though it lacks a truthmaker at that world since its truth at that world is a brute fact of it. The other is that our intuition here tracks what we can know about the truth or falsity of if prime, odd at worlds like $w^{*}$ - this allows us to say that if prime, odd is either true or false at $w^{*}$, we just don't know which. Both are possible avenues worth exploring - for instance, see Khoo 2013 for an epistemicist implementation of the semantics in $\S 1.3$ that rejects (CI-1) in this latter way. In this paper, I want to focus on a different avenue that allows us to hold on to (CI-1).
} 
tence as uttered in that context is true at those worlds, hence our second premise (assuming that the existential quantifier here is restricted to epistemically accessible worlds):

$$
\mathrm{P}_{c}(\mathrm{p})>0 \text { only if } \exists w: \mathrm{p} \text { is true at } c, w .
$$

Sentential probability seems to entail (CI-2), given the assumption that propositions are true or false relative to worlds. However, there is independent motivation for (CI-2) as well. Our interest in sentential probability is probability of truth, so given the assumption that sentences are true or false relative to worlds (and contexts), there must be some world at which the sentence is true for it to have a nonzero probability of truth.

With (CI-1) and (CI-2) in hand, the problem of indeterminacy follows straightforwardly. From (CI-1) we know there are some Ramseyan, A-indeterminate, A-uncertain conditional/context pairs. Let $\langle\ulcorner$ if p, $\mathrm{q}\urcorner, c\rangle$ be such a pair. From (CI-2) it follows that $\operatorname{Pr}_{c}(\ulcorner$ if $\mathrm{p}, \mathrm{q}\urcorner \mid$ not-p $)=0$. Substituting this into the law of total probability:

$$
\operatorname{Pr}_{c}(\ulcorner\text { if } \mathrm{p}, \mathrm{q}\urcorner)=\operatorname{Pr}_{c}(\ulcorner\text { if } \mathrm{p}, \mathrm{q}\urcorner \mid \mathrm{p}) \cdot \operatorname{Pr}_{c}(\mathrm{p})+\operatorname{Pr}_{c}(\ulcorner\text { if } \mathrm{p}, \mathrm{q}\urcorner \mid \text { not-p }) \cdot \operatorname{Pr}_{c}(\text { not-p })
$$

yields:

$$
\operatorname{Pr}_{c}(\ulcorner\text { if } \mathrm{p}, \mathrm{q}\urcorner)=\operatorname{Pr}_{c}(\ulcorner\text { if } \mathrm{p}, \mathrm{q}\urcorner \mid \mathrm{p}) \cdot \operatorname{Pr}_{c}(\mathrm{p})
$$

Finally, recall PC:

$$
\text { PC: } \operatorname{Pr}_{c}(\ulcorner\text { if p, q }\urcorner \mid \mathrm{p})=\operatorname{Pr}_{c}(\mathrm{q} \mid \mathrm{p}) \quad \text { (For every } \mathrm{p}, \mathrm{q},\ulcorner\text { if } \mathrm{p}, \mathrm{q}\urcorner \text {, and } c \text {.) }
$$

Hence, substituting into (14) yields:

$$
\operatorname{Pr}_{c}(\ulcorner\text { if } \mathrm{p}, \mathrm{q}\urcorner)=\operatorname{Pr}_{c}(\mathrm{q} \mid \mathrm{p}) \cdot \operatorname{Pr}_{c}(\mathrm{p})
$$

But now since $\langle\ulcorner$ if p, $\mathrm{q}\urcorner, c\rangle$ is Ramseyan it follows that:

$$
\operatorname{Pr}_{c}(\mathrm{q} \mid \mathrm{p})=\operatorname{Pr}_{c}(\mathrm{q} \mid \mathrm{p}) \cdot \operatorname{Pr}_{c}(\mathrm{p})
$$

And since $\langle\ulcorner$ if $\mathrm{p}, \mathrm{q}\urcorner, c\rangle$ is A-uncertain, we have a contradiction. Hence, we have a contradiction that follows from three plausible claims: (CI-1), PC, and Sentential probability (which seems to entail (CI-2)). Thus, the three are jointly inconsistent - this is the problem of indeterminacy.

I pause to mention four ways out of this problem that I will set aside for now. First, we might deny (CI-1) on the grounds that conditionals like (6) are true at some $\neg$ prime- 
worlds - see for instance the discussion in fn. 14. I think this is a promising approach, but I want to confine our discussion here to ways of handling the problem that allow us to hold on to (CI-1) (see Khoo 2013 for a way to implement this strategy in a way analogous to the approach I take in $\S 1.3$ ). The second way out is to deny (CI-1) by denying that conditional/context pairs like $\left\langle(6), c_{1}\right\rangle$ are Ramseyan - this is the approach favored by those who deny Ramsey's observation. ${ }^{15}$ However, such views fly directly in the face of our intuitions, and hence face the challenge of accounting for why it seems so plausible that $\langle(6)$, $\left.c_{1}\right\rangle$ is Ramseyan. Third, we might deny PC. However, the discussion in $\S 1.1$ shows that the principle is well-motivated, and thus that giving it up would be a significant cost. Finally, we could reject Sentential probability and hence undercut some of the motivation for (CI-2). However, although a non-propositionalist who denies Sentential probability need not be committed to (CI-2), her account is incomplete without some account of the sense in which conditional sentences have probabilities if they aren't probabilities of truth, and this is a tall order. ${ }^{16}$

Unlike the above four responses, the response I favor is conservative - I aim to hold on to both Sentential probability and Ramsey's observation and yet deny (CI-2) by motivating the claim that a sentence $\mathrm{p}$ can have a nonzero probability of truth in a context $c$ even though there is no epistemically accessible world in $c$ relative to which $\mathrm{p}$ is (determinately) true. In the next section I'll sketch a propositionalist semantics for indicatives that predicts exactly this result.

\subsection{Semantics}

In this section, I'll sketch an orthodox propositionalist semantics for indicative conditionals with a twist that will allow us to avoid the problem of indeterminacy by denying (CI-2). Thus, one-half of the moral of the story for indicative conditionals and the work of $\S 1.3-1.4$ is to motivate a propositionalist semantics for indicative conditionals in which they can be neither true nor false at each $\neg p$-world and also have a nonzero probability throughout the

\footnotetext{
${ }^{15}$ We need not give up Ramsey's observation to adopt such an approach, but the trouble for this strategy remains the same.

${ }^{16}$ For instance, we might hold that what's to be explained is not the probability that some conditional sentence is true but rather the acceptability (or degree of belief) we have in such sentences, and then hold that it is a fundamental feature of conditional sentences that their acceptability (or the degree of belief we have in them) goes by the Ramseyan Thesis (see for instance Adams 1975, Edgington 1995, Bennett 2003). However, such a view runs into trouble predicting the non-Ramseyan conditional/context pairs discussed in McGee 2000, Kaufmann 2004. Without a plausible story about those- how is it that a brute fact can have exceptions? - this sort of primitivist view is in rough shape.
} 
$\neg p$-region of logical space.

We begin with a baseline semantic framework in which 【』 is a function from expressions to extensions (truth values, in the case of sentences) relative to a context $c$ and world $w$. We'll let our truth values be 1 and 0 ; thus, $\llbracket \mathrm{p} \rrbracket^{c, w}=1 / 0$ iff $\mathrm{p}$ is true/false at $c, w$. From here, we define the intension of a sentence at a context to be a function from worlds to truth values (the sentence's extension at that context and world); hence, the intension of $\mathrm{p}$ at $c=p=\llbracket \mathrm{p} \rrbracket^{c}=\left\{w: \llbracket \mathrm{p} \rrbracket^{c, w}=1\right\}$. I'll adopt the usual doublespeak and use the expression "the proposition expressed by $\mathrm{p}$ at $c$ " to refer both to the intension of $\mathrm{p}$ at $c$ and the set of possible worlds for which that intension is a characteristic function.

Here's an initial statement of the proposed truth conditions for indicative conditionals:

$$
\llbracket\ulcorner\text { if } \mathrm{p}, \mathrm{q}\urcorner \rrbracket^{c, w}=1 \text { iff the closest epistemically accessible }{ }_{c, w} p \text {-world is a } q \text {-world. }
$$

Spelling out these truth conditions requires that we say more about epistemic accessibility and the closeness relations on worlds. We model epistemic accessibility by way of a function $E$ from worlds to sets of worlds - those that are compatible with what is known at the input world. Thus, at the very least, $E(w)$ must be factive:

$$
\text { Factivity: } \forall w: w \in E(w) \text {. }
$$

We'll also assume that each context $c$ determines a unique epistemic accessibility relation $E_{c}$ (this might correspond to what's compatible, for each $w$, with what is known at $w$ by those members of the conversation occurring at $c$ - for our purposes, the value of $E_{c}$ doesn't particularly matter). Thus, the epistemically accessible ${ }_{c, w}$ worlds are just the worlds in $E_{c}(w)$.

We characterize the closeness relation in two parts:

- Limit: For any context $c$ and worlds $w$ and $w^{\prime} \in E_{c}(w)$ : if $p$ is true at $w^{\prime}$, then $w^{\prime}$ is the closest $p$-world to $w^{\prime}$ in $E_{c}(w)$.

- Similar: For any context $c$ and worlds $w$ and $w^{\prime} \in E_{c}(w)$ : if $p$ is false at $w^{\prime}$, then the closest $p$-world to $w^{\prime}$ in $E_{c}(w)$ is among the most relevantly similar $p$-worlds to $w^{\prime}$ in $E_{c}(w)$.

Limit says that if $p$ is true at $w$, then $\llbracket\ulcorner$ if $\mathrm{p}, \mathrm{q}\urcorner \rrbracket^{c, w}=1$ iff $\llbracket \mathrm{q} \rrbracket^{c, w}=1$. Thus, by Limit we predict that $\mathbf{P C}$ holds. ${ }^{17}$ Similar requires a bit more explanation - in particular, what

\footnotetext{
${ }^{17}$ We assume Sentential probability. Then $\operatorname{Pr}_{c}(\ulcorner$ if $\mathrm{p}, \mathrm{q}\urcorner \mid \mathrm{p})=P_{c}($ if $p, q \mid p)$. By Limit, if $p, q$ is
} 
are the most relevantly similar $p$-worlds to $w^{\prime}$ in $E_{c}(w)$ ? To answer this, we must say what facts of $w^{\prime}$ count toward relevant similarity with respect to $w^{\prime}$. One fruitful way to model this is to let relevant similarity be fixed by a partition on $E_{c}(w), Z .{ }^{18}$ Let $[w]_{Z}$ denote the cell of $Z$ in which $w$ falls. The basic idea is that it is the $Z$-features of $w$ that count towards a world being relevantly similar to $w$. Or, putting it more picturesquely, the most similar worlds to $w$ given $Z$ are $[w]_{Z}$. Thus, the most similar $p$-worlds to $w$ are $[w]_{Z} \wedge p$. Plugging this into Similar yields:

- Similar*: For any context $c$ and worlds $w$ and $w^{\prime} \in E_{c}(w)$, and where $Z$ is the relevance partition for $\ulcorner$ if $\mathrm{p}, \mathrm{q}\urcorner$ in $c$, the closest $p$-world to $w^{\prime}$ in $E_{c}(w)$ is among $\left[w^{\prime}\right]_{Z} \wedge p$.

I want to set aside the difficult question of how a relevance partition for a conditional is determined in a context, ${ }^{19}$ except to note that we need a constraint to avoid the following problem: if $Z$ is a relevance partition for $\ulcorner$ if $\mathrm{p}, \mathrm{q}\urcorner$ in $c$ and $w$ is such that there are no $p$-worlds in $[w]_{Z}$, then the extension of $\ulcorner$ if $\mathrm{p}, \mathrm{q}\urcorner$ (as uttered in $c$ ) will be undefined at $w$. I assume that this situation is to be avoided - intuitively, only those features of $w$ that are compatible with $p$ need to be held fixed in determining the closest $p$-world to $w$. Thus, we stipulate that $Z$ is an admissible relevance partition for $\ulcorner$ if $\mathrm{p}, \mathrm{q}\urcorner$ in $c$ only if each cell $z \in Z$ is compatible with $E_{c}(w)$ and $p$, that is, only if there is some $z \wedge p$-world $w^{\prime}$ in $E_{c}(w)$, for each $z \in Z$, that is, only if $Z$ is $p$-compatible.

Similar* gives us a constraint on closeness when $p$ is false at $w$, but doesn't determine a unique closest $p$-world to $w$ in such a case unless there is a single world in $[w]_{Z} \wedge p$. Thus, since Similar* is the only constraint on closeness when $p$ is false, if there is more than one world in $[w]_{Z} \wedge p$, our semantics leaves it indeterminate which among them is the closest $p$-world to $w$ (again, assuming $p$ is false at $w$ ). It is how we understand and incorporate this indeterminacy into our semantic framework that will be critical to solving the indeterminacy problem.

equivalent to $q$ at the $p$-worlds. Hence, we may substitute $q$ for if $p, q$ in the previous equation, yielding: $\operatorname{Pr}_{c}(\ulcorner$ if $\mathrm{p}, \mathrm{q}\urcorner \mid \mathrm{p})={ }_{c} P(q \mid p)$, which entails PC given Sentential probability.

${ }^{18} \mathrm{~A}$ partition on a set of worlds $W$ is a set of subsets of those worlds $Z$ such that $\bigcap Z=W$ and for which every $w \in W$ : there is exactly one $z \in Z$ such that $w \in z$. Less formally, $Z$ is a set of boxes of worlds of $W$ such that every world in $W$ is in at least one box in $Z$, and no world in any box of $Z$ is in any other box in $Z$.

${ }^{19}$ See Khoo 2013 for a positive proposal about the features of context that determine relevance partitions. 


\subsection{Avoiding the indeterminacy problem}

Before we begin, I should mention that the approach to indeterminacy I sketch here is not forced upon us - similar results (and analogous solutions to the challenge from indeterminacy) can be obtained via different theories of indeterminacy (see Khoo 2013 for discussion). The approach we'll adopt understands the indeterminacy in closest $p$-world to be metaphysical in nature - as there being an objective chance about which $p$-world is closest, even if there is no objective fact about which actually is closest. ${ }^{20}$ Another way of putting the idea is that the truth values of indicative conditionals are sensitive to distinctions that cut more finely than distinctions between possible worlds - so, although possible worlds represent all the determinate distinctions between possibilities that can be drawn, further distinctions that give rise to (metaphysical) indeterminacies may be drawn as well.

To fix some terminology, let's call these world-like entities that draw distinctions more finely than possible worlds precisifiers. To model the relationship between worlds and precifiers, we'll assume that precisifiers are basic and worlds are sets of precisifiers. Thus, we have both a set of all worlds $W$ and a set of all precisifiers $F$ such that $F=\cup W$. Precifiers will now play the role in our semantics that worlds did previously, in defining intensions for instance; we'll then define new relations of determinately true, determinately false, and indeterminate between sentential intensions and worlds. ${ }^{21}$ Thus, $\llbracket$ is now a function from expressions to extensions relative to a context $c$ and precisifier $f$. The intension of $\mathrm{p}$ at $c=\llbracket \mathrm{p} \rrbracket^{c}=\left\{f: \llbracket \mathrm{p} \rrbracket^{c, f}=1\right\}$. Since they play exactly the role in our new theory as propositions played in our old theory, it will do no harm to call these precisifierintensions propositions (sets of precisifiers are as much truth conditions as sets of possible worlds, only sensitive to distinctions more fine-grained than can be drawn between possible worlds). Thus, propositions are now true/false relative to precisifiers, whereas they are determinately true/false relative to worlds:

$$
\llbracket p \rrbracket^{c} \text { is }
$$

\footnotetext{
${ }^{20}$ Inspirations for this approach include Akiba 2004, Barnes and Williams 2011, Barnes and Cameron 2011 and the following insightful quote from Stalnaker 1984 (though he's talking about counterfactuals and our topic is indicatives): "The selection functions relative to which counterfactuals are interpreted do not simply select on the basis of facts and criteria of similarity that are intelligible independently of counterfactuals. Rather, the claim is, the fact of selection gives rise to new ways of cutting up the space of possibilities, and so to a richer conception of the way the world is" (169).

${ }^{21}$ The ramifications of these definitions will be many. For instance, possible worlds are ways things might determinately be, while precisifiers are simply ways things might be. Furthermore, it may be indeterminate which precifisier we are at, although it will be determinate which world we are at. And so on.
} 

a. $\quad$ determinately true at $w$ if $\forall f \in w: \llbracket p \rrbracket^{c, f}=1$
b. determinately true at $w$ if $\forall f \in w: \llbracket p \rrbracket^{c, f}=0$
c. indeterminate at $w$ otherwise

Intuitively, on this understanding of indeterminacy, determinate truth/falsity just is having objective chance $1 / 0$, and indeterminacy is having an objective chance not equal to 0 or 1 . Say that the objective chance of $p$ relative to $w$ is equal to the proportion of precisifers in $w$ at which $p$ is true. Thus, on this theory, there can be two worlds $w_{1}$ and $w_{2}$ which agree on the truth value of all the determinate propositions but differ in the chance they assign to some proposition that is indeterminate at at least one of them.

Applying our theory to Similar* requires us first to restate our semantics for $\ulcorner$ if $\mathrm{p}$, $\mathrm{q}\urcorner$ at the level of precisifiers:

$$
\begin{aligned}
& \llbracket\ulcorner\text { if } \mathrm{p}, \mathrm{q}\urcorner \rrbracket^{c, f}=1 \text { iff } \forall f^{\prime} \in w: \llbracket \mathrm{q} \rrbracket^{c, f^{\prime}}=1 \text {; where } w \text { is the closest } p \text {-world to } f \text { among } \\
& E_{c}\left(w^{f}\right) .
\end{aligned}
$$

Two remarks: the truth of the conditional requires the determinate truth of $q$ at the closest $p$-world, and epistemic accessibility is still a relation on worlds $-w^{f}$ is the world of $f$. Both of these might be subject to revision, depending on how we may we want to handle indeterminacy within the consequents of conditionals, but for the sake of space let's set that matter aside for now and plow ahead. ${ }^{22}$ We revise Limit and Similar* at the level of precisifiers (this is where our approach to indeterminacy gets teeth):

- Limit-M: For any context $c$ and worlds $w$ and $w^{\prime} \in E_{c}(w)$ : if $p$ is determinately true at $w^{\prime}$, then $\forall f \in w^{\prime}: w^{\prime}$ is the closest $p$-world to $f$ in $E_{c}(w)$.

- Similar-M: For any context $c$ and worlds $w$ and $w^{\prime} \in E_{c}(w)$ and where $Z$ is the relevance partition for $\ulcorner$ if $\mathrm{p}, \mathrm{q}\urcorner$ in $c$ : if $p$ is determinately false at $w^{\prime}$, then for each $f \in w^{\prime}$ : there is a unique world $w^{\prime \prime} \in\left[w^{\prime}\right]_{Z} \wedge p$ such that $w^{\prime \prime}$ is the closest $p$-world to $f$ in $E_{c}(w)$.

Similar-M ensures that for each $\neg p$-world $w^{\prime}$, "closest $p$-world" behaves as a one-to-one function from precisifiers $f \in w^{\prime}$ to worlds in $\left[w^{\prime}\right]_{Z} \wedge p$. It does so by filtering the precisifiers making up each $\neg p$-world, demanding that they conform to this constraint. In effect, what

\footnotetext{
${ }^{22}$ Furthermore, the division of labor in stating our revised Limit and Similar principles is split between $p$ being determinately true at $w^{\prime}$ and determinately false at $w^{\prime}$-we leave it open for now what happens when $p$ is indeterminate at $w^{\prime}$.
} 
it does is translate the information about what worlds comprise $\left[w^{\prime}\right]_{Z}$ into information about the chance, at each $\neg p$-world in $w^{\prime}$, what the closest $p$-world to it is. Thus, Similar$\mathbf{M}$ has no effect on the truth values of any determinate propositions, but it may affect the chances of indeterminate propositions. And, in turn, we can appeal to it to predict non-trivial probabilities for sentences that are indeterminate. Let $\operatorname{ch}(p)(w)$ be the chance of $p$ at $w$ and $\mathcal{C}(p)(z)$ be the average chance of $p$ throughout the worlds in $z$. Then, we'll say that ordinary probability of a proposition just is the expectation of the chance that $p$, given each member of a set of propositions that jointly partition the epistemic space:

$$
P_{c}(p)=\sum_{z \in Z} \mathcal{C}(p)(z) \cdot P_{c}(z) \quad \text { Where } Z \text { partitions } E_{c}(w) .
$$

Take some arbitrary sentence $\mathrm{p}$ that expresses the proposition $p$ (at $c$ ) such that $p$ is indeterminate (and hence neither determinately true nor determinately false) at each world in some region $z$. Nonetheless, as long as its chance is nonzero at some world $w \in z$ such that $P_{c}(z)>0$, it will be the case that $\operatorname{Pr}_{c}(\mathrm{p})>0$. Thus, by endorsing this semantics we reject (CI-2) - a sentence may fail to be determinately true (relative to $c$ ) at every world in $E_{c}(w)$ and yet the probability that that sentence is true may still be nonzero. ${ }^{23}$ Furthermore, our semantics predicts Prob, which allows us to predict not only that $\operatorname{Pr}_{c}(\ulcorner$ if $\mathrm{p}, \mathrm{q}\urcorner \mid$ not-p) is nontrivial but also the right value for it in various contexts: ${ }^{24}$

\footnotetext{
${ }^{23}$ To be completely precise, by endorsing this semantics we reject (CI-2) as stated because we reject that sentences are true or false relative to contexts and worlds - instead holding that they are true/false relative to contexts and precisifiers. The principle the semantics falsifies is the corresponding version of (CI-2) with determinate truth:

$\left(\mathrm{CI}-2^{*}\right) \operatorname{Pr}_{c}(\mathrm{p})>0$ only if $\exists w: \mathrm{p}$ is determinately true at $c, w$.

${ }^{24}$ Here's the derivation:

$$
\begin{array}{llr}
\text { a. } & P_{c}(\text { if } p, q \mid \neg p)= & \text { Sentential probability } \\
\text { b. } & \sum_{z \in Z} \mathcal{C}(\text { if } p, q)(z \wedge \neg p) \cdot P_{c}(z \mid \neg p)= & \text { Def. } \mathcal{C} \\
\text { c. } & \sum_{z \in Z} P_{c}(q \mid z \wedge p) \cdot P_{c}(z \mid \neg p)= & \text { See }(*) \text { below }
\end{array}
$$$$
\operatorname{Pr}_{c}(\ulcorner\text { if } \mathrm{p}, \mathrm{q}\urcorner \mid \text { not-p })=
$$

$\left(^{*}\right)$ Here's why $\mathcal{C}$ (if $\left.p, q\right)(z \wedge \neg p)=P_{c}(q \mid z \wedge p)$. Take an arbitrary $z \wedge \neg p$-world $w^{\prime} \in E_{c}(w)$. By Similar-M, for each $w^{\prime \prime} \in z \wedge p$, there is exactly one precisifier $f \in w^{\prime}$, such that the closest $p$-world to $f$ is $w^{\prime \prime}$. And since $\llbracket\ulcorner$ if $\mathrm{p}, \mathrm{q}\urcorner \rrbracket^{c, f}=1$ iff $q$ is determinately true at $w$, where $w$ is the closest $p$-world to $f$, it follows that the proportion of precisifiers $f \in w^{\prime}$ at which $\llbracket\ulcorner$ if $\mathrm{p}, \mathrm{q}\urcorner \rrbracket^{c, f}=1$ is equal to the proportion of $q$-worlds in $z \wedge p$. Hence, $\operatorname{ch}($ if $p, q)(w)=P_{c}(q \mid z \wedge p)$. But the choice of $z \wedge \neg p$-world was arbitrary, so this holds for every $z \wedge \neg p$-world. Therefore, the average chance that if $p, q$ throughout the worlds in $z \wedge \neg p$ is equal to the probability that $q$ given $z \wedge p$, that is, $\mathcal{C}($ if $p, q)(z \wedge \neg p)=P(q \mid z \wedge p)$.
} 
Prob: $\operatorname{Pr}_{c}(\ulcorner$ if $\mathrm{p}, \mathrm{q}\urcorner \mid$ not-p $)=\sum_{z \in Z} P_{c}(q \mid z \wedge p) \cdot P_{c}(z \mid \neg p)$

This semantics is designed to predict both Ramseyan and non-Ramseyan conditional/context pairs ${ }^{25}$ but for now let's focus on how it avoids the problem of indeterminacy by predicting both Sentential probability and (CI-1). Let $\langle\ulcorner$ if p, q $\urcorner, c\rangle$ be such that $Z_{c}=\left\{E_{c}(w)\right\}$. Suppose also that $p \wedge q, p \wedge \neg q$ and $\neg p$ are compatible with $E_{c}(w)$, and let $w^{\prime}$ be an arbitrary $\neg p$-world in $E_{c}(w)$. Then our semantics predicts that $\llbracket\ulcorner$ if $\mathrm{p}, \mathrm{q}\urcorner \rrbracket^{c}$ is indeterminate at $w^{\prime} .^{26}$ Nonetheless, our semantics predicts that $\operatorname{Pr}_{c}(\ulcorner$ if $\mathrm{p}, \mathrm{q}\urcorner)=P_{c}(q \mid p) .{ }^{27}$ Therefore, we predict that $\langle\ulcorner$ if $\mathrm{p}, \mathrm{q}\urcorner, c\rangle$ is Ramseyan, A-indeterminate, and A-uncertain, and hence (CI-1), and we do so while also predicting Sentential probability.

\section{Problems of triviality}

We turn now to the problems for Sentential probability and Ramsey's observation that stem from the various triviality results. In stating these problems, it will be helpful to have the property of being an R-function (R for "Ramsey") for a conditional/context pair:

For any $\langle\ulcorner$ if $\mathrm{p}, \mathrm{q}\urcorner, c\rangle: P$ is an R-function for $\langle\ulcorner$ if $\mathrm{p}, \mathrm{q}\urcorner, c\rangle$ iff $P($ if $p, q)=P(q \mid p)$.

\footnotetext{
${ }^{25}$ It predicts Ramseyan conditional/context pairs in the following condition (where $Z$ is the relevance partition on $\ulcorner$ if $\mathrm{p}, \mathrm{q}\urcorner$ in $c$ ):$$
\operatorname{Pr}_{c}(\ulcorner\text { if } \mathrm{p}, \mathrm{q}\urcorner)=\operatorname{Pr}_{c}(\mathrm{q} \mid \mathrm{p}) \text { iff } \sum_{z \in Z} P_{c}(q \mid z \wedge p) \cdot P_{c}(z \mid \neg p)=P_{c}(q \mid p)
$$

See Khoo 2013 for more detailed discussion of this result and how our semantics predicts which conditionals are intuitively Ramseyan in which contexts.

${ }^{26}$ Since $Z_{c}=\left\{E_{c}(w)\right\},\left[w^{\prime}\right]_{Z}=E_{c}(w)$. Thus, $\left[w^{\prime}\right]_{Z} \wedge p$ contains both $q$ and $\neg q$-worlds (by the assumption that $p \wedge q$ and $p \wedge \neg q$ are both compatible with $\left.E_{c}(w)\right)$. Therefore, by Similar-M, there is a $f \in w^{\prime}$ such that the closest $p$-world to $f$ in $E_{c}(w)$ is a $q$-world, which ensures that $\llbracket\ulcorner$ if $\mathrm{p}, \mathrm{q}\urcorner \rrbracket^{c, f}=1$. And also by Similar-M, there is a $f^{\prime} \in w^{\prime}$ such that closest $p$-world to $f^{\prime}$ in $E_{c}(w)$ is a $\neg q$-world, which ensures that $\llbracket\ulcorner$ if $\mathrm{p}, \mathrm{q}\urcorner \rrbracket^{c, f^{\prime}}=0$. Putting both together, we predict that $\llbracket\ulcorner$ if $\mathrm{p}, \mathrm{q}\urcorner \rrbracket^{c}$ is indeterminate at $w^{\prime}$.

${ }^{27}$ Here's the calculation:

$$
\begin{array}{llr}
\operatorname{Pr}_{c}(\ulcorner\text { if } \mathrm{p}, \mathrm{q}\urcorner)= & \text { Total probability } \\
\text { a. } & \operatorname{Pr}_{c}(\ulcorner\text { if } \mathrm{p}, \mathrm{q}\urcorner \mid \mathrm{p}) \cdot \operatorname{Pr}_{c}(\mathrm{p})+\operatorname{Pr}_{c}(\ulcorner\text { if } \mathrm{p}, \mathrm{q}\urcorner \mid \text { not-p }) \cdot \operatorname{Pr}_{c}(\text { not-p }) & \text { PC, Sentential probability, Prob } \\
\text { b. } & P_{c}(q \mid p) \cdot P_{c}(p)+\sum_{z \in Z_{c}} P_{c}(q \mid z \wedge p) \cdot P_{c}(z \mid \neg p) \cdot P_{c}(\neg p) & \text { Since } Z_{c}=\left\{E_{c}(w)\right\} \\
\text { c. } & P_{c}(q \mid p) \cdot P_{c}(p)+P_{c}(q \mid p) \cdot P_{c}(\neg p) & \text { Algebra } \\
\text { d. } & P_{c}(q \mid p) &
\end{array}
$$$$
\text { b. } \quad P_{c}(q \mid p) \cdot P_{c}(p)+\sum_{z \in Z_{c}} P_{c}(q \mid z \wedge p) \cdot P_{c}(z \mid \neg p) \cdot P_{c}(\neg p) \quad \text { PC, Sentential probability, Prob }
$$ 
(Where if $p, q, p$, and $q$ are the propositions expressed by the sentences $\ulcorner$ if $\mathrm{p}$, $\mathrm{q}\urcorner$, p, and $\mathrm{q}$ in $c$-I'll adopt this convention throughout.)

With this new terminology in hand, we can state the following consequence of Sentential probability and Ramsey's observation:

SR: There are conditional/context pairs $\langle\ulcorner$ if p, $\mathrm{q}\urcorner, c\rangle$ such that $P_{c}$ is an R-function for $\langle\ulcorner$ if $\mathrm{p}, \mathrm{q}\urcorner, c\rangle$.

The various triviality proofs establish (in different senses) that not every probability function is an R-function for every indicative in every context. ${ }^{28}$ Triviality proofs come in two kinds: some establish that the property of being an R-function for $\langle\ulcorner$ if $p, q\urcorner, c\rangle$ does not hold across a class of probability functions (inter-function EQ failures), and others that, holding fixed a particular probability function $P$ and context $c$, there will always be conditional/context pairs whose context-coordinate is $c$ for which $P$ is not an R-function (intra-function EQ failures). In the following sections, I will discuss both kinds of triviality proofs and argue that neither raises trouble for combining Sentential probability and Ramsey's observation, since the bridge principles necessary to generate an inconsistency with SR are plausibly false.

\subsection{Inter-function proofs}

Here, we'll consider the class of triviality proofs which aim to show that, for any $\langle\ulcorner$ if $\mathrm{p}$, $\mathrm{q}\urcorner, c\rangle$ : the property of being an R-function for $\langle\ulcorner$ if $\mathrm{p}, \mathrm{q}\urcorner, c\rangle$ does not hold across some privileged class of probability functions. ${ }^{29}$ We'll review as the representative of such proofs Hájek's version of Lewis's original triviality result (Hájek 2011b). ${ }^{30}$ Let's begin with a piece of terminology: let $P^{\mid x}$ be the probability function that results from conditionalizing $P$ on $x$ :

\footnotetext{
${ }^{28}$ We are restricting our attention to conditionals that express propositions in the context under discussion. Hence, we'll set aside cases where $P$ is not an R-function for $\langle\ulcorner$ if $\mathrm{p}, \mathrm{q}\urcorner, c\rangle$ because $\ulcorner$ if $\mathrm{p}$, $\mathrm{q}\urcorner$ does not express any proposition in $c$.

${ }^{29}$ For instance, Lewis 1976, 1986, Carlstrom and Hill 1978.

${ }^{30}$ I discuss Hájek's generalization rather than Lewis's original proof (Lewis 1976) because any semantics that predicts that if $p, q$ can be true at some $\neg p \wedge \neg q$-world can falsify one of the assumptions of Lewis's proof (that $P($ if $p, q \mid \neg q)=P(q \mid p \cap \neg q)=0$ ) - see Rothschild 2011 for discussion-but Hájek's generalization does not rely on this assumption. Furthermore, Hájek proves that the same result generalizes beyond conditionalizing to any revision rule that is "bold" and "moderate" (properties shared by imaging, maxent, minxent, and various blurred imagings, among others - see Hájek 2011b for a full discussion).
} 


$$
\forall p, q \in \wp(W): P^{\mid p}(q)=_{\text {def }} P(q \mid p), \text { where } P(p)>0
$$

A class of probability functions $\mathbb{C}$ is closed under conditionalizing iff any probability function that results from conditionalizing some function in $\mathbb{C}$ on some proposition is in $\mathbb{C}$. Let $\mathbb{C}_{P}$ be the class of probability functions that contains $P$ and is closed under conditionalizing. Say that a probability function $P$ is not $\mathrm{H}$-trivial for $\langle\ulcorner$ if $\mathrm{p}, \mathrm{q}\urcorner, c\rangle$ iff $P(p)<1 \wedge P(p \wedge q)>0 \wedge P(p \wedge \neg q)>0$. The conclusion of Hájek's proof is:

Hájek's Result: for any $P$ that is an R-function for $\langle\ulcorner$ if p, q $\urcorner, c\rangle$ and also not H-trivial for $\langle\ulcorner$ if $\mathrm{p}, \mathrm{q}\urcorner, c\rangle$, there will be some probability function $P^{\prime} \in \mathbb{C}_{P}$ such that $P^{\prime}$ is not an R-function for $\langle\ulcorner$ if p, q $\urcorner, c\rangle$.

A bit more simply, Hájek's Result is that the property of being a non H-trivial Rfunction for $\langle\ulcorner$ if $\mathrm{p}, \mathrm{q}\urcorner, c\rangle$ does not hold across the class of probability functions closed under conditionalizing. Since it would be an intolerable result that the only R-functions for $\langle\ulcorner$ if p, $\mathrm{q}\urcorner, c\rangle$ are ones that are H-trivial for $\langle\ulcorner$ if $\mathrm{p}, \mathrm{q}\urcorner, c\rangle$, we can take it that Hájek's Result establishes:

Hájek's Conclusion: if $P$ is an R-function for $\langle\ulcorner$ if $\mathrm{p}, \mathrm{q}\urcorner, c\rangle$, then there will be some probability function $P^{\prime} \in \mathbb{C}_{P}$ such that $P^{\prime}$ is not an R-function for $\langle\ulcorner$ if p, q $\urcorner$, $c\rangle$.

This is not yet enough to yield a contradiction when paired with Sentential probability and Ramsey's observation. The minimal principle needed is:

H-Bridge: If $P$ is an R-function for $\langle\ulcorner$ if $\mathrm{p}, \mathrm{q}\urcorner, c\rangle$, then every probability function $P^{\prime} \in \mathbb{C}_{P}$ is such that $P^{\prime}$ is an R-function for $\langle\ulcorner$ if $\mathrm{p}, \mathrm{q}\urcorner, c\rangle$.

H-Bridge says, in effect, that if you fix on the proposition expressed by $\ulcorner$ if $\mathrm{p}, \mathrm{q}\urcorner$ in $c$, call it $x$, and a probability function $P$ such that $P(x)=P(q \mid p)$, then every probability function $P^{\prime}$ related to $P$ by conditionalization will be such that $P^{\prime}(x)=P^{\prime}(q \mid p)$. From $\mathbf{S R}$ we have that $P_{c}$ is an R-function for $\langle\ulcorner$ if $\mathrm{p}, \mathrm{q}\urcorner, c\rangle$, which together with H-Bridge entails that every $P \in \mathbb{C}_{P_{c}}$ is an R-function for $\langle\ulcorner$ if p, $\mathrm{q}\urcorner, c\rangle$. But from Hájek's Conclusion it follows that there is some probability function $P^{\prime} \in \mathbb{C}_{P_{c}}$ such that $P^{\prime}$ is not an R-function for $\langle\ulcorner$ if $\mathrm{p}, \mathrm{q}\urcorner, c\rangle$ - thus, we have a contradiction. 
In what follows, I'll present a simplified version of Hájek's elegant proof. ${ }^{31}$ SR entails that there are conditional/context pairs $\langle\ulcorner$ if p, q $\urcorner, c\rangle$ such that $P_{c}$ is a non-H-trivial EQ function for $\langle\ulcorner$ if p, q $\urcorner, c\rangle$ (the extra assumption that $P_{c}$ is non-H-trivial should be uncontroversial given examples like $\left\langle(6), c_{1}\right\rangle$ from $\left.\S 1.2\right)$. I'll adopt the following shorthand, writing $P^{\mid x}$ for the probability function that results from conditionalizing $P$ on $x$ : hence, $P^{\mid x}(z)=P(z \mid x)$. For simplicity of illustration, assume that $p$ and $q$ are independent and both have probability 0.5. Given this, plus PC, it follows that if $p, q$ is true throughout the $p \wedge q$ region of epistemic space $\left(E_{c}(w)\right)$ and false throughout the $p \wedge \neg q$ region, and has some probability distributed throughout the $\neg p$ region such that $P_{c}($ if $p, q \mid \neg p)=P_{c}($ if $p, q \mid p)$. So, things are as in Figure 1.

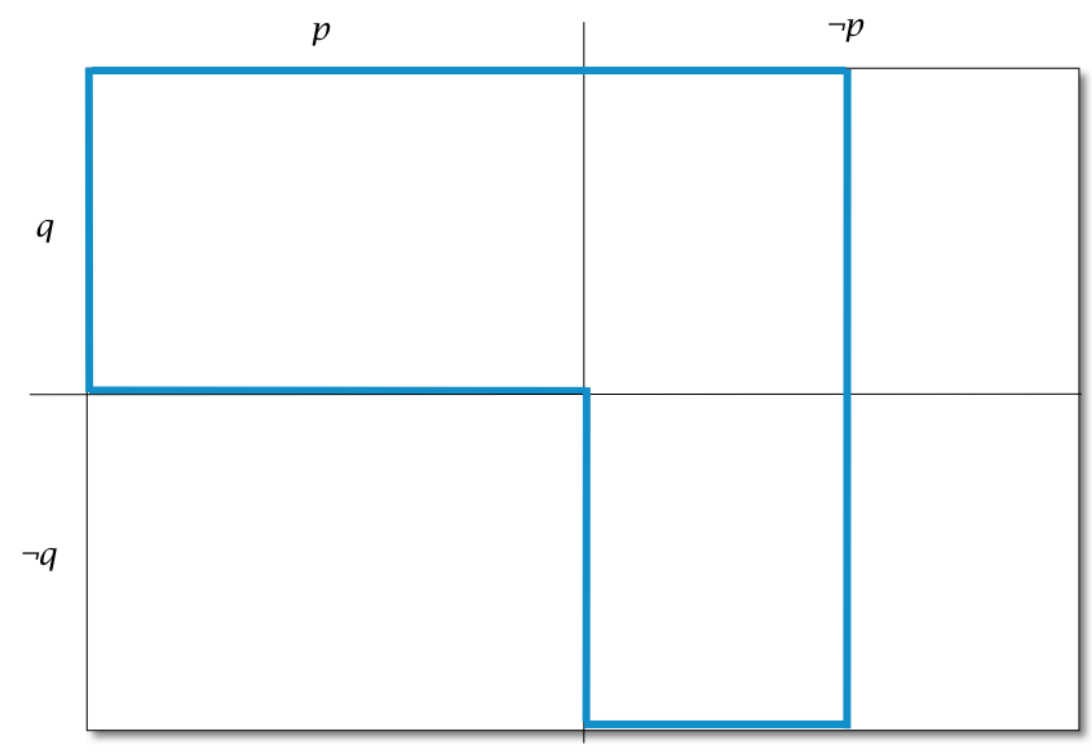

Figure 1

Now, by conditionalizing $P_{c}$ on $z$-that is $\neg(p \wedge q)$-we eliminate the $p \wedge q$-worlds, and renormalize over the remaining worlds. But now notice that it's obvious that $P_{c}^{\mid z}(q \mid p)=0$, since the only $p$-worlds that are left are all $\neg q$-worlds. But at the same time, there's still some probability mass of if $p, q$ distributed throughout the $\neg p$-worlds, and hence $P_{c}^{\mid z}($ if $p, q) \neq 0$. Thus, $\mathbf{P C}$ and $\mathbf{S R}$ entail that $\mathbf{H}$-Bridge is false; or, alternatively, $\mathbf{H}-$ Bridge entails that either $\mathbf{P C}$ or $\mathbf{S R}$ is false (and hence that $\mathbf{S R}$ is false, since we're

\footnotetext{
${ }^{31}$ Those readers interested in the formal proof should consult Hájek 2011b, pp. 10-12.
} 
assuming $\mathbf{P C}$ is true). Thus, holding fixed $\mathbf{P C}$, it follows that either $\mathbf{S R}$ or $\mathbf{H}$-Bridge is false. But which should we give up?

I contend that $\mathbf{S R}$ is more plausible than $\mathbf{H}$-Bridge and hence we ought to reject the latter. To motivate this claim, let's consider a concrete scenario analogous to the one constructed in our informal version of Hájek's proof. John has just rolled a fair six-sided die and kept the result hidden - call this context $c$ and suppose that (6) is uttered in $c$ :

(6) If John rolled a prime, then he rolled an odd.

Let prime and odd denote the propositions that John rolled a prime and that John rolled an odd. Notice that both prime/ $\neg$ prime and odd/ $\neg$ odd are compatible with what is known in $c$. Let if ${ }_{c}$ prime, odd be the proposition expressed by the utterance of (6) in $c$, let $c^{+}$be the context exactly like $c$ except that it's known in $c^{+}$that $\neg($ prime $\wedge$ odd $)$. Finally, notice that $P_{c^{+}}($odd $\mid$prime $)=0$, since there are no prime $\wedge$ odd-worlds compatible with what's known in $c^{+}$. What is at issue is whether $P_{c^{+}}\left(\right.$if ${ }_{c}$ prime, odd $)=P_{c^{+}}($odd $\mid$prime $)=0$ that is, whether the probability, once we've learned $\neg($ prime $\wedge$ odd $)$, of the proposition we expressed before learning that relevant fact by uttering (6) equals 0 . Now, let's turn to two arguments that $P_{c^{+}}($if $c$ prime, odd $) \neq 0$.

Argument 1. Suppose you say (6) prior to learning anything about the roll. Then you learn that John didn't both roll a prime and an odd. Given your new evidence, there are still possibilities in which what you said (if $f_{c}$ prime, odd) is definitely falsethese are the prime $\wedge \neg$ odd possibilities (e.g., where he rolled a 2). But what about the $\neg$ prime-possibilities compatible with your new evidence (e.g., where he rolled a 1, 4, or

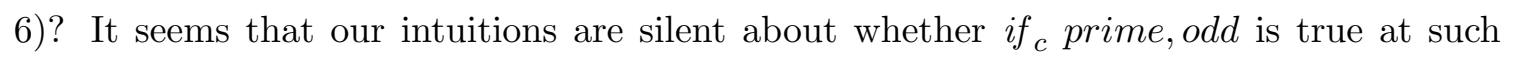
worlds - hence, it seems wrong to conclude that what you said is certainly false at them. Furthermore, our discussion of the problem of indeterminacy from $\S 1$ cautioned against such a conclusion. Therefore, it seems plausible that if $f_{c}$ prime, odd has some non-zero probability throughout the $\neg$ prime region of logical space, and this is reason to reject that that $P_{c^{+}}\left(\right.$if $f_{c}$ prime, odd $)=0$.

Argument 2. $\forall x: P_{c^{+}}(x)=P_{c}(x \mid \neg($ prime $\wedge$ odd $))$, so if $P_{c^{+}}\left(\right.$if $f_{c}$ prime, odd $)=0$ then $P_{c}\left(\right.$ if $f_{c}$ prime,$o d d \mid \neg($ prime $\wedge$ odd $\left.)\right)=0$. But this entails that if $f_{c}$ prime, odd is equivalent to prime $\wedge$ odd, which is false - it is intuitively weaker than its corresponding conjunction. 
Hence, it seems that we do not judge that what you said when you uttered (6) in the prior context has a probability of 0 after learning that John didn't both roll a prime and an odd. If this is correct, then we have a counterexample to H-Bridge that is structurally identical to the conditionalization-case constructed in Hájek's triviality proof.

These two arguments aim to bring out the unintuitive character of $\mathbf{H}$-Bridge in light of SR. Thus, they are only as compelling as our reasons for holding SR. And we might be persuaded to reject $\mathbf{S R}$ if we had a compelling reason to endorse $\mathbf{H}$-Bridge. I turn to an argument for $\mathbf{H}$-Bridge now. I'll argue that we can plausibly reject $\mathbf{H}$-Bridge even in light of this argument.

An argument for H-Bridge. Suppose after learning that John didn't roll a prime and an odd, you rehearse the following argument:

(23) i. Given what I now know, either John didn't roll a prime or he didn't roll an odd.

ii. So, it must be that if John rolled a prime, then he didn't roll an odd.

iii. Therefore, (6) must be false (by conditional non-contradiction).

Thus, we have an apparently sound argument whose conclusion seems to be that the probability that (6) is true is 0 - just what $\mathbf{H}$-Bridge predicts about the situation constructed in Hájek's proof. What should we say about this argument? First, we must be slightly more careful. Simply because the probability that the sentence (6) is true is 0 after having learned $\neg($ prime $\wedge$ odd $)$ does not mean that the proposition expressed by that sentence in the previous context has zero probability after having learned that evidence. After all, if what proposition an indicative conditional expresses depends on the possibilities compatible with the available evidence in the context, then the proposition expressed by (6) in the original context (prior to learning $\neg($ prime $\wedge$ odd)) and the proposition expressed by (6) in the posterior context (after having learned $\neg($ prime $\wedge$ odd $)$ ) may be different.

In fact, this is exactly what our semantics from $\S 1.3$ predicts. In what follows, I'll sketch a quick example to illustrate why, for two contexts with distinct evidence $c$ and $c^{*}$, our semantics predicts that the probability (given the evidence in $c^{*}$ ) of the proposition expressed by $\ulcorner$ if $\mathrm{p}, \mathrm{q}\urcorner$ in $c$ may be different from the probability (given the evidence in $c^{*}$ ) of the proposition expressed by $\ulcorner$ if $\mathrm{p}, \mathrm{q}\urcorner$ in $c^{*}$. As before, let $E_{c}(w)$ be the set of worlds compatible with the evidence in $c, w, P_{c}$ be the probability function associated with $c-$ 
defined over $E_{c}(w)$ - and let if $c$ p, $q$ be the proposition expressed by $\ulcorner$ if $\mathrm{p}, \mathrm{q}\urcorner$ in $c$. Suppose that $E_{c}(w)=\left\{w, w_{1}, w_{2}\right\}$ and suppose that the closest $p$-world to $w, w_{1}$ and $w_{2}$ is $w_{1}$, and that $w_{1}$ is the only $q$-world in $E_{c}(w)$. Then, the proposition expressed by $\ulcorner$ if $\mathrm{p}, \mathrm{q}\urcorner$ at $c$ is $\left\{w, w_{1}, w_{2}\right\}$ and hence $P_{c}\left(i f_{c} p, q\right)=1$. Now, suppose that $E_{c^{*}}(w)=\left\{w, w_{2}\right\}$. Basically, $c^{*}$ is related to $c$ by learning something that rules out $q$. Since there are no $q$-worlds in $E_{c^{*}}(w)$, none of the closest worlds to any world in $E_{c^{*}}(w)$ is a $q$-world, so the proposition expressed by $\ulcorner$ if $\mathrm{p}, \mathrm{q}\urcorner$ at $c^{*}$ is $\emptyset$. Hence, $P_{c^{*}}\left(i f_{c^{*}} p, q\right)=0$. But of course, the proposition expressed by $\ulcorner$ if $\mathrm{p}, \mathrm{q}\urcorner$ is different in $c$ than in $c^{*}$, and hence it may not be the case that the probability (given the evidence available in $c^{*}$ ) of the proposition expressed by $\ulcorner$ if $\mathrm{p}$, $\mathrm{q}\urcorner$ in $c$ is 0 . In fact, in this situation, the probability (given the evidence available in $c^{*}$ ) of that proposition is still 1 - that is, $P_{c^{*}}\left(\right.$ if $\left.f_{c} p, q\right)=1$.

Now, our semantics can predict both what is appealing about the argument from (i)(iii) and our intuition that the probability (given what is known in $c^{+}$) of the proposition expressed by the utterance of (6) in $c$ is not zero. We predict that the step from (i) to (ii) in the argument is valid-once you're in a context in which it's taken for granted that $\neg($ prime $\wedge$ odd $)$ then the proposition expressed by (6) in this context will have a probability 0 and its negation will have probability 1 . But the step from (ii) to (iii) will be invalid if we understand it as a judgment about the probability that the sentence (6) in the prior context is true, and valid only if we understand it as a judgment about the probability that (6) in the posterior context is true. Thus, the kind of context-dependent semantics motivated in $\S 1.3$ can predict both intuitions without appealing to H-Bridgetherefore, we are warranted in rejecting $\mathbf{H}$-Bridge on the grounds that it is falsified in the scenario constructed in Hájek's proof, given PC and SR. ${ }^{32}$ An exhaustive defense of context-dependent semantics for indicative conditionals is beyond the scope of this paper, and thus since I cannot find any additional arguments for H-Bridge, I conclude that we are warranted in thinking that $\mathbf{H}$-Bridge is false.

\footnotetext{
${ }^{32}$ To put the point another way: our context-dependent propositionalist semantic has the resources to account for the intuitions that seem to support H-Bridge-for instance, the appeal of arguments like (i)-(iii) - without H-Bridge being true. It does so by ensuring that whenever there is a match between the context $c$ relative to which the indicative expresses a proposition and the context that sets the available evidence relevant for calculating probabilities, then, setting aside cases of independence failure, $P_{c}$ will be an R-function for $\langle\ulcorner$ if $\mathrm{p}, \mathrm{q}\urcorner, c\rangle$. Someone looking to defend H-Bridge must either try to undermine this kind of context-dependent semantics, or find some additional argument for $\mathbf{H}$-Bridge that is not amenable to this kind of plausible counter-explanation.
} 


\section{$2.2 \quad$ Intra-function proofs}

Inter-function triviality proofs are only a problem for $\mathbf{S R}$ given a suitable bridge principle. In $\S 2.1$ we saw that a plausible way to avoid such problems is to reject $\mathbf{H}$-Bridge rather than SR, thus preserving both Sentential probability and Ramsey's observation. In this section, we'll consider two intra-function triviality proofs which establish that, holding fixed the probability function $P$ and context $c$, there will always be some conditional $\ulcorner$ if $\mathrm{p}$, $\mathrm{q}\urcorner$ such that $P$ is not an R-function for $\langle\ulcorner$ if $\mathrm{p}, \mathrm{q}\urcorner, c\rangle$. Going through these proofs is crucial for a defense of $\mathbf{S R}$, since the problems they raise are not avoided by a context-sensitive semantics, but will instead showcase the features of our semantics that allowed us to avoid the problem of indeterminacy. I'll discuss the proofs of Robert Stalnaker (Stalnaker 1976) and Alan Hájek (Hájek 1989, 2012) in $§ 2.2 .1$ and 2.2.2 below.

\subsubsection{Stalnaker's proof}

The conclusion of Stalnaker's proof is:

Stalnaker's Result: for any $P$ that is an R-function for $\langle\ulcorner$ if $\mathrm{p}, \mathrm{q}\urcorner, c\rangle$, there is another conditional $\ulcorner$ if $\mathrm{r}, \mathrm{s}\urcorner$ such that $P$ is not an R-function for $\langle\ulcorner$ if $\mathrm{r}, \mathrm{s}\urcorner, c\rangle$.

There is no contradiction between Stalnaker's Result and SR. To yield a contradiction, we need at least the following bridge principle:

S-Bridge: for any conditional/context pairs $\langle\ulcorner$ if p, $\mathrm{q}\urcorner, c\rangle$ and $\langle\ulcorner$ if $\mathrm{r}, \mathrm{s}\urcorner, c\rangle$ and any $P$ that is an R-function for $\langle\ulcorner$ if $\mathrm{p}, \mathrm{q}\urcorner, c\rangle, P$ is an R-function for $\langle\ulcorner$ if $\mathrm{r}, \mathrm{s}\urcorner, c\rangle$.

S-Bridge is a generality principle, just like $\mathbf{H}$-Bridge; basically, it states that, once you've got an R-function for some conditional in some context, that probability function is also an R-function for every other conditional in that context. From SR we have some conditional/context pair such that $P_{c}$ is an R-function for $\langle\ulcorner$ if $\mathrm{p}, \mathrm{q}\urcorner, c\rangle$, and then this and S-Bridge together entail that for any conditional $\ulcorner$ if $\mathrm{x}, \mathrm{y}\urcorner, P_{c}$ is also an R-function for $\langle\ulcorner$ if $\mathrm{x}, \mathrm{y}\urcorner, c\rangle$. But then Stalnaker's result entails that $P_{c}$ is not an R-function for some conditional/context pair $\langle\ulcorner$ if $\mathrm{r}, \mathrm{s}\urcorner, c\rangle$-thus, we have a contradiction.

I'll discuss S-Bridge momentarily. Our proof of Stalnaker's Result proceeds by construction (my presentation here follows Edgington 1995). Fix our context $c$ throughout, and focus on the following propositions (as expressed by their corresponding sentences at c): 
- Let $x$ be $\neg p \wedge($ if $p, q)$.

- Let $r$ be $\neg x$.

- Let $s$ be $p \wedge \neg q$.

Suppose our probabilities given $P_{c}$ for these propositions are partitioned into four regions (which we may treat as "worlds") as follows:

\begin{tabular}{c|c|c|c|c|c|c|c|c}
$w$ & $P_{c}(\{w\})$ & $p$ & $q$ & if $p, q$ & $r$ & $s$ & $x$ & if $r, s$ \\
\hline 1 & 0.25 & $T$ & $T$ & $T$ & $T$ & $F$ & $F$ & $F$ \\
2 & 0.25 & $T$ & $F$ & $F$ & $T$ & $T$ & $F$ & $T$ \\
3 & 0.25 & $F$ & & $T$ & $F$ & $F$ & $T$ & $F$ \\
4 & 0.25 & $F$ & & $F$ & $T$ & $F$ & $F$ & $F$
\end{tabular}

We suppose that if $p, q$ has this distribution of truth/falsity throughout the $\neg p$-region to ensure that $P_{c}$ (if $\left.p, q\right)=P_{c}(q \mid p)$. Given these propositions, if $r, s$ is false at $w_{1}$ and $w_{4}$ because $r \wedge \neg s$ hold there, and if $r, s$ is true at $w_{2}$ because $r \wedge s$ holds there - this holds because indicatives are strongly centered (as our semantics entails). Why is if $r, s$ is false at $w_{3}$ ? The reason is that $x$ entails that if $r, s$ is false. This is so because $x$ entails if $p, q$, but if $r, s$ entails $\neg($ if $p, q) .{ }^{33}$ Hence, $x$ entails $\neg($ if $r, s)$, and thus that if $r, s$ is false at $w_{3}$. But now notice that $P_{c}($ if $r, s) \neq P_{c}(s \mid r)$, for $P_{c}(s \mid r)=\frac{1 / 4}{3 / 4}=1 / 3$, while $P_{c}($ if $r, s)=1 / 4$. Hence, we have established Stalnaker's Result, since the choice of $P$ and conditional were arbtirary.

I accept Stalnaker's Result. ${ }^{34}$ Thus, Stalnaker's Result entails that one of either SR or S-Bridge is false. Here's an argument that we ought to take Stalnaker's Result as evidence against $\mathbf{S}$-Bridge rather than $\mathbf{S R}$. Recall that, given $\mathbf{P C}$, any $\langle\ulcorner$ if $\mathrm{p}, \mathrm{q}\urcorner, c\rangle$ which is not antecedent independent is such that $P_{c}$ is not an R-function for $\langle\ulcorner$ if $\mathrm{p}, \mathrm{q}\urcorner$, $c\rangle$. That is, if $P_{c}$ (if $\left.p, q \mid p\right) \neq P_{c}($ if $p, q)$, then $P_{c}($ if $p, q) \neq P_{c}(q \mid p)$. But now notice that $\langle\ulcorner$ if $\mathrm{r}, \mathrm{s}\urcorner, c\rangle$ is not antecedent independent- that is, $P_{c}($ if $r, s) \neq P_{c}($ if $r, s \mid r)$. The latter value is, unsurprisingly, $1 / 3$, while the former is $1 / 4$. Thus, far from establishing a problem

\footnotetext{
${ }^{33}$ Suppose if $r, s$ is true. if $r, s$ is true at $w$ iff the closest $r$-world to $w$ is an $s$ world iff the closest $p \vee \neg($ if $p, q)$-world to $w$ is a $p \wedge \neg q$-world. Let $w^{\prime}$ be the closest $p \vee \neg($ if $p, q)$-world to $w$. Since if $r, s$ is true at $w$ (by hypothesis), $w^{\prime}$ is a $p \wedge \neg q$-world. $w^{\prime}$ is either a $p$-world or a $\neg p$-world. Suppose $w^{\prime}$ is a $\neg p$-world. Then $w^{\prime}$ is a $p \wedge \neg p$-world, which cannot be the case. Therefore, $w^{\prime}$ must be a $p$-world. But then the closest $p$-world to $w$ is a $\neg q$-world, in which case $\neg($ if $p, q)$ is true at $w$.

${ }^{34}$ Though see Bennett 2003, p. 73 for some ways of rejecting this conclusion.
} 
for propositionalism about indicatives, Stalnaker's proof merely establishes that there are ways of spelling out a context $c$ such that, even though $P_{c}$ is an R-function for $\langle\ulcorner$ if $\mathrm{p}$, $\mathrm{q}\urcorner, c\rangle$, there is another conditional $\ulcorner$ if $\mathrm{r}, \mathrm{s}\urcorner$, such that $\langle\ulcorner$ if $\mathrm{r}, \mathrm{s}\urcorner, c\rangle$ that is not antecedent independent. And if this is true, then given PC, S-Bridge must be false. This may be surprising, but it is not a problem, and is perfectly compatible with $\mathbf{S R}$.

Before we go on, I want to show how our semantics from $\S 1$ predicts this result. It turns out that our semantics requires that, in the context sketched in the course of the proof, there must be an admissible non-trivial relevance partition for $\ulcorner$ if $r, s\urcorner$. But what relevance partition will bring about the right results? A natural candidate is $\{p, \neg p\}$. First, notice that this is an admissible relevance partition for $\ulcorner$ if $\mathrm{r}, \mathrm{s}\urcorner$ in $c$, since $r \wedge p$ is true at $w_{1}$ and $r \wedge \neg p$ is true at $w_{4}$. Next, recall from $\S 1.2$ that $P_{c}($ if $r, s \mid r) \neq P_{c}$ (if $\left.r, s\right)$ iff $P_{c}($ if $r, s \mid r) \neq P_{c}$ (if $\left.r, s \mid \neg r\right)$. Hence, we must calculate $P_{c}($ if $r, s \mid \neg r)$ and to this we turn to Prob* (which follows from Prob given Sentential probability):

$$
\text { Prob*: } P_{c}(\text { if } r, s \mid \neg r)=\sum_{z \in Z} P_{c}(s \mid z \wedge r) \cdot P_{c}(z \mid \neg r)
$$

Thus:

- $P_{c}($ if $r, s \mid \neg r)=$

$$
\begin{aligned}
& P_{c}(s \mid p \wedge r) \cdot P_{c}(p \mid \neg r)+P_{c}(s \mid \neg p \wedge r) \cdot P_{c}(\neg p \mid \neg r)= \\
& 1 / 2 \cdot 0+0 \cdot 1=0
\end{aligned}
$$

Hence, $P_{c}($ if $r, s \mid \neg r)=0$. But $P_{c}($ if $r, s \mid r)=P_{c}(s \mid r)=1 / 3$, by PC. Hence, $P_{c}($ if $r, s) \neq$ $P_{c}(s \mid r)$. Thus, as long as $\ulcorner$ if $\mathrm{r}, \mathrm{s}\urcorner$ receives $\{p, \neg p\}$ as its relevance partition in $c$, our semantics predicts exactly the situation constructed in the proof. Thus, we can take the lesson of Stalnaker's proof to be that the mere fact that $P_{c}$ is an R-function for $\langle\ulcorner$ if $\mathrm{p}, \mathrm{q}\urcorner$, $c\rangle$ does not guarantee, for every conditional $\ulcorner$ if $\mathrm{x}, \mathrm{y}\urcorner$, that $P_{c}$ will be an R-function for $\langle\ulcorner$ if $\mathrm{x}, \mathrm{y}\urcorner, c\rangle$.

\subsubsection{Hájek's wallflower proof}

Alan Hájek’s "wallflower" proof (Hájek 1989, 1994, 2012) establishes the following: ${ }^{35}$

\footnotetext{
${ }^{35}$ So-called because there must be some wallflowers - conditional probability values for $P$ — not paired with any unconditional probability value for $P$. Here's a simplified version of the proof from Hájek 2012. Suppose $W=\left\{w_{1}, w_{2}, w_{3}\right\}$ and that $P$ is a probability function defined over $W$ such that $P\left(\left\{w_{1}\right\}\right)=$
} 
Wallflower Result: any non-trivial finite-ranged probability function $P$ has more distinct conditional probability values than distinct unconditional probability values.

What's meant by non-trivial here is that $P$ is defined over a finite set of worlds $W$ such that $|W|>2$. As it stands, the Wallflower Result is not incompatible with $\mathbf{S R}$, for all it ensures is that, for any context $c$, if $P_{c}$ is a non-trivial finite-ranged probability function, then there are conditionals $\ulcorner$ if $\mathrm{p}, \mathrm{q}\urcorner$ such that $P_{c}$ is not an R-function for $\langle\ulcorner$ if $\mathrm{p}, \mathrm{q}\urcorner, c\rangle$. And, in our discussion of Stalnaker's Result we saw exactly how this might happensuch a result is obtained if $\ulcorner$ if $\mathrm{p}, \mathrm{q}\urcorner$ has a relevance partition in $c$ that makes $\langle\ulcorner$ if $\mathrm{p}, \mathrm{q}\urcorner, c\rangle$ not antecedent independent.

However, although the Wallflower Result is strictly compatible with our semantics, it threatens the following very plausible assumption:

Trivial Relevance: it is possible that there are contexts $c$ such that every conditional $\ulcorner$ if $\mathrm{p}, \mathrm{q}\urcorner$ is assigned a trivial relevance partition $\left(=\left\{E_{c}(w)\right\}\right)$ in $c$.

This seems plausible, and if it is true, then given our semantics, there must be contexts $c$ for which it is possible that, for every conditional $\ulcorner$ if $\mathrm{p}, \mathrm{q}\urcorner, P_{c}$ is an R-function for $\langle\ulcorner$ if $\mathrm{p}, \mathrm{q}\urcorner, c\rangle$. But this is what the Wallflower Result says cannot be the case. The Wallflower Result thus complicates any picture of how relevance partitions are assigned to conditionals in context, raising doubts for the sort of work our semantics aims to put relevance partitions to use - in particular, threatening to undermine a natural explanation of non-Ramseyan conditional/context pairs (cf. Kaufmann 2004, Khoo 2013). Therefore, it would be nice to have a way of blocking the upshot of the Wallflower Result for our semantics.

I'll offer two plausible strategies for blocking the upshot of the Wallflower Result for our semantics. The first is to reject Trivial Relevance. Recall that a relevance partition $Z$ for $\ulcorner$ if $\mathrm{p}, \mathrm{q}\urcorner$ interacts with Similar-M to place a constraint on the precisifiers making up each $\neg p$-world $w^{\prime} \in E_{c}(w)$-namely, demanding that they can be put in one-to-one correspondence with the worlds in $\left[w^{\prime}\right]_{Z} \wedge p$. However, if there are not enough precisifiers

$P\left(\left\{w_{2}\right\}\right)=P\left(\left\{w_{3}\right\}\right)=1 / 3$. The probability of any proposition $p \in \wp(W)$ must then be a multiple of $1 / 3$. However, various conditional probabilities are not multiples of $1 / 3$. Let $p=\left\{w_{1}, w_{2}\right\}$ and $q=\left\{w_{1}\right\}$. Then, $P(q \mid p)=\frac{P\left(\left\{w_{1}\right\}\right)}{P\left(\left\{w_{1}, w_{2}\right\}\right)}=\frac{1 / 3}{2 / 3}=1 / 2$. Therefore, there are conditional probability values for $P$ that do not equal unconditional probability values for $P$. Hájek 1989 proves that this result generalizes for any finite-ranged probability function. 
in in some $w^{\prime}$, then this cannot be done. If the set of precisifiers in $\cup E_{c}(w)$ is finite, by the Wallflower Result we know that Trivial Relevance must be false. As far as I can tell, accepting this consequence as entailed by the Wallflower Result is not problematic for our semantics. The prediction that there are no contexts in which every conditional is assigned a trivial relevance partition is not a reductio of our theory. After all, it doesn't seem unreasonable to hold that no ordinary speech context will determine a relevance partition for every conditional sentence, in which case our theory won't make any false predictions about ordinary speech contexts. Indeed, we might even conjecture that the kind of context that could determine a relevance partition for every conditional sentence differs so much from the ordinary speech contexts we are acquainted with that it's no shortcoming of a semantic/pragmatic theory that such contexts fall outside of its purview.

On the other hand, if we are motivated to accept Trivial Relevance, then by the Wallflower Result we must conclude that the range of $P_{c}$ is infinite for those possible contexts $c$ in which every conditional $\ulcorner$ if $\mathrm{p}, \mathrm{q}\urcorner$ gets a trivial relevance partition in $c$ (call these trivial contexts). This result has seemed strange to some - after all, it has the consequence that any agent (including ideally rationally agents) will be such that any probability function $P_{c}$ modeling their credences in a trivial context $c$ is such that the range of $P_{c}$ is infinite. ${ }^{36}$ Restrict attention to agents whose credences in $c$ are modeled by probability functions that are R-functions for the class of Ramseyan conditional/context pairs whose context-coordinate is $c$, and suppose the domain of such functions is the set of possibleworlds propositions; then, the result entails that the domain of any agent's probability function is always infinite. This has the strange consequence that even ideally rational agents (including those lacking constraints of memory or time to gather information) must be uncertain of an infinite number of propositions. Furthermore, even if we are prepared to accept such a result for other reasons, it is striking that it is forced upon us on the basis of linguistic considerations and some probability theory.

Nonetheless, I think we can tell a reasonable story about why such a consequence would arise from the indeterminacy of conditionals within our theory. Recall that in our framework, we handled this indeterminacy within a framework that models propositions as sets of precisifiers $(F)$ rather than possible worlds $(W)$, and thus the domain of $P$ is $\wp(F)$ rather than $\wp(W)$, such that $p$ is indeterminate at $w$ iff $0<\operatorname{ch}(p)(w)<1$. Given this,

\footnotetext{
${ }^{36}$ Cf. Hájek and Hall 1994 (p. 98), who note that "it is difficult to see how, in addition, rationality would preclude the appropriateness of finite or less-than-full functions (particularly when considering the highly idealized agent)."
} 
the consequence that every agent (including ideally rational agents) must be uncertain of an infinite number of propositions is secured by the additional stipulation that (i) any set of possible worlds $E$ representing the credal space of some agent is such that there is an infinite set of metaphysically indeterminate propositions $\mathcal{S} \subseteq \wp(\cup E) .{ }^{37}$ This stipulation seems reasonable on at least two grounds. One, we've introduced the technology of precisifiers for the express purpose of modeling the indeterminacy of conditionals, so we should be prepared to endorse whatever features of $F$ are mathematically needed for them to do their job. And two, in making this assumption we still allow for the possibility of rational agents who are certain of all the determinate propositions, which softens some of the initial strangeness of the result. Therefore, even if we think Trivial Relevance is plausible, we can tell a reasonably plausible story about the kind of consequences it gives rise to, given the Wallflower Result. Thus, finally, no matter what our attitude toward Trivial Relevance, our semantics is insulated from trouble arising from Hájek's Wallflower Result.

\section{Conclusion}

The problems raised by indeterminacy and triviality complicate the most straightforward relationship between the truth conditions of indicative conditionals and their probabilities. In the course of this paper, I hope to have shown that such problems do not force us to give up either of the plausible principles we began with-Sentential probability and Ramsey's observation. On the contrary, the problems reveal two crucial features of indicative conditionals that any semantic theory of conditionals ought to take seriously. The first is that indicatives are often indeterminate in such a way that they fail to be (determinately) true at some worlds and yet have a non-zero probability throughout those worlds, and the second that what proposition indicatives express crucially depends on the set of possible worlds that are epistemically possible in the context. Recognizing these properties of indicatives, my semantics showed one natural and conservative way to handle the problems of indeterminacy and triviality. I hope this paper adds an additional notch in the belt of such a semantics, and also provides some illumination as to the often-unclear upshots of these various problems for the project of theorizing about the semantics of

\footnotetext{
${ }^{37} \mathrm{By}$ our rule of calculating probabilities, $P(p)=\sum_{w \in W} \operatorname{ch}(p)(w)$. And if for every $p \in \mathcal{S}, \exists w \in W: 0<$ $\operatorname{ch}(p)(w)<1$, this ensures that for every $p \in \mathcal{S}: 0<P(p)<1$.
} 
indicative conditionals. ${ }^{38}$

\section{References}

Adams, E. (1975). The Logic of Conditionals, Volume 86 of Synthese Library. Boston: D. Reidel.

Adams, E. (1998). A Primer of Probability Logic. Stanford: CSLI.

Akiba, K. (2004). Vagueness in the world. Nous 38(3), 407-429.

Barnes, E. and R. P. Cameron (2011). Back to the open future. Philosophical Perspectives 25, 1-26.

Barnes, E. and J. R. G. Williams (2011). A theory of metaphysical indeterminacy. In Oxford Studies in Metaphysics. Oxford: Oxford University Press.

Bennett, J. (2003). A Philosophical Guide to Conditionals. Oxford: Oxford University Press.

Carlstrom, I. and C. Hill (1978). Review of adams's the logic of conditionals. Philosophy of Science 45, 155-158.

Cross, C. (2009). Conditional excluded middle. Erkenntnis 70, 173-188.

DeRose, K. (2002). Assertion, knowledge, and context. The Philosophical Review 111(2), $167-203$.

Edgington, D. (1995). On conditionals. Mind 104, 235-329.

von Fintel, K. and S. Iatridou (2002). If and when if-clauses can restrict quantifiers. http://web.mit.edu/fintel/www/lpw.mich.pdf.

Gibbard, A. (1981). Two recent theories of conditionals. In W. L. Harper, R. Stalnaker, and G. Pearce (Eds.), Ifs, pp. 211-247. Dordrecht: Reidel.

Grice, P. (1989). Studies in the Way of Words. Cambridge: Harvard University Press.

Hájek, A. (1989). Probabilities of conditionals - revisited. Journal of Philosophical Logic 18, 423-428.

\footnotetext{
${ }^{38} \mathrm{My}$ thinking on this topic has benefited from conversations and comments from many people. Audiences at Johns Hopkins, MIT, UMass, and Yale provided helpful suggestions on an earlier version of this work. I'd also like to thank Laura Beavers, Phil Bricker, Dan Greco, Larry Horn, Mark Maxwell, Vann McGee, Chris Meacham, Aaron Norby, Bob Stalnaker, Zoltán Szabó, Steve Yablo, and Bruno Whittle for helpful comments.
} 
Hájek, A. (1994). Triviality on the cheap? In E. Eells and B. Skyrms (Eds.), Probability and Conditionals: Belief Revision and Rational Decision, pp. 113-140. Cambridge: Cambridge University Press.

Hájek, A. (2003). What conditional probability could not be. Synthese 137(3), 273-323.

Hájek, A. (2011a). Most counterfactuals are false. ms.

Hájek, A. (2011b). Triviality pursuit. Topoi 30, 3-15.

Hájek, A. (2012). The fall of "adams' thesis"? Journal of Logic, Language, and Information 21, 145-161.

Hájek, A. and N. Hall (1994). The hypothesis of the conditional construal of conditional probability. In E. Eells and B. Skyrms (Eds.), Probability and Conditionals, Chapter 6, pp. 75-111. Cambridge: Cambridge University Press.

Higginbotham, J. (1986). Truth and Interpretation: Perspectives on the Philosophy of Donald Davidson, Chapter Linguistic Theory and Davidson's Program in Semantics, pp. 29-48. Oxford: Blackwell.

Higginbotham, J. (2003). Conditionals and compositionality. Philosophical Perspectives: Language and Philosophical Linguistics 17, 181-194.

Horn, L. (1985). Metalinguistic negation and pragmatic ambiguity. Language 61(1), 121174.

Jackson, F. (1979). On assertion and indicative conditionals. Philosophical Review 88, $565-89$.

Kaufmann, S. (2004). Conditioning against the grain: Abduction and indicative conditionals. Journal of Philosophical Logic 33, 583-606.

Kaufmann, S. (2005). Conditional predictions. Linguistics and Philosophy 28, 181-231.

Khoo, J. (2013). Probability of conditionals in context. ms.

Klinedinst, N. (2010). Quantified conditionals and conditional excluded middle. Journal of Semantics 27(3), 1-22.

Lewis, D. (1973). Counterfactuals. Oxford: Blackwell.

Lewis, D. (1976). Probabilities of conditionals and conditional probabilities. The Philosophical Review 85, 297-315.

Lewis, D. (1986, October). Probabilities of conditionals and conditional probabilities ii. The Philosophical Review 95, 581-589. 
McGee, V. (2000). To tell the truth about conditionals. Analysis 60(1), 107-111.

Moss, S. (2012). Subjunctive credences. Philosophy and Phenomenological Research.

Ramsey, F. P. (1931). The Foundations of Mathematics and other Logical Essays. London: Kegan Paul, Trench, Trubner \& Co.

Rothschild, D. (2011). Do indicative conditionals express propositions? Nous.

Stalnaker, R. (1976). Letter to van fraassen. In W. Harper and C. Hooker (Eds.), Foundations of Probability Theory, Statistical Inference, and Statistical Theories of Science, Volume 1, pp. 302-306. Dordrecht: Reidel.

Stalnaker, R. (1980). A defense of conditional excluded middle. In W. L. Harper, G. Pearce, and R. Stalnaker (Eds.), Ifs. Dordrecht: Reidel.

Stalnaker, R. (1984). Inquiry. MIT Press.

Williams, J. R. G. (2010). Defending conditional excluded middle. Nous 44(4), 650-668.

Williamson, T. (1996). Knowing and asserting. The Philosophical Review 105(4), 489-523. 